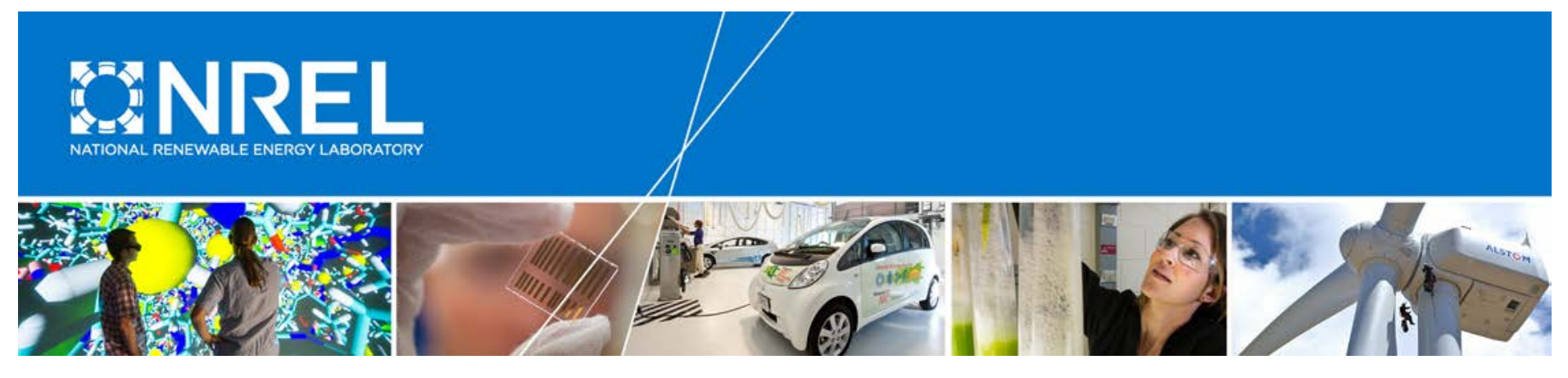

\title{
Short-Term Load Forecasting Based Automatic Distribution Network Reconfiguration
}

\section{Preprint}

Huaiguang Jiang, Fei Ding, and Yingchen Zhang National Renewable Energy Laboratory

Presented at the 2017 IEEE Power \& Energy Society General Meeting (PES GM)

Chicago, Illinois

July 16-20, 2017

(C) 2017 IEEE. Personal use of this material is permitted. Permission from IEEE must be obtained for all other uses, in any current or future media, including reprinting/republishing this material for advertising or promotional purposes, creating new collective works, for resale or redistribution to servers or lists, or reuse of any copyrighted component of this work in other works.

NREL is a national laboratory of the U.S. Department of Energy Office of Energy Efficiency \& Renewable Energy Operated by the Alliance for Sustainable Energy, LLC

This report is available at no cost from the National Renewable Energy Laboratory (NREL) at www.nrel.gov/publications.

\section{Conference Paper}

NREL/CP-5D00-67762

July 2017 


\section{NOTICE}

The submitted manuscript has been offered by an employee of the Alliance for Sustainable Energy, LLC (Alliance), a contractor of the US Government under Contract No. DE-AC36-08GO28308. Accordingly, the US Government and Alliance retain a nonexclusive royalty-free license to publish or reproduce the published form of this contribution, or allow others to do so, for US Government purposes.

This report was prepared as an account of work sponsored by an agency of the United States government. Neither the United States government nor any agency thereof, nor any of their employees, makes any warranty, express or implied, or assumes any legal liability or responsibility for the accuracy, completeness, or usefulness of any information, apparatus, product, or process disclosed, or represents that its use would not infringe privately owned rights. Reference herein to any specific commercial product, process, or service by trade name, trademark, manufacturer, or otherwise does not necessarily constitute or imply its endorsement, recommendation, or favoring by the United States government or any agency thereof. The views and opinions of authors expressed herein do not necessarily state or reflect those of the United States government or any agency thereof.

This report is available at no cost from the National Renewable Energy Laboratory (NREL) at www.nrel.gov/publications.

Available electronically at SciTech Connect http:/www.osti.gov/scitech

Available for a processing fee to U.S. Department of Energy and its contractors, in paper, from:

U.S. Department of Energy

Office of Scientific and Technical Information

P.O. Box 62

Oak Ridge, TN 37831-0062

OSTI http://www.osti.gov

Phone: 865.576.8401

Fax: 865.576.5728

Email: reports@osti.gov

Available for sale to the public, in paper, from:

U.S. Department of Commerce

National Technical Information Service

5301 Shawnee Road

Alexandria, VA 22312

NTIS http://www.ntis.gov

Phone: 800.553 .6847 or 703.605 .6000

Fax: 703.605.6900

Email: orders@ntis.gov 


\title{
Short-Term Load Forecasting Based Automatic Distribution Network Reconfiguration
}

\author{
Huaiguang Jiang, Member, IEEE, Fei Ding, Member, IEEE, and Yingchen Zhang, Senior Member, IEEE
}

\begin{abstract}
In a traditional dynamic network reconfiguration study, the optimal topology is determined at every scheduled time point by using the real load data measured at that time. The development of the load forecasting technique can provide an accurate prediction of the load power that will happen in a future time and provide more information about load changes. With the inclusion of load forecasting, the optimal topology can be determined based on the predicted load conditions during a longer time period instead of using a snapshot of the load at the time when the reconfiguration happens; thus, the distribution system operator can use this information to better operate the system reconfiguration and achieve optimal solutions. This paper proposes a short-term load forecasting approach to automatically reconfigure distribution systems in a dynamic and pre-event manner. Specifically, a short-term and high-resolution distribution system load forecasting approach is proposed with a forecaster based on support vector regression and parallel parameters optimization. The network reconfiguration problem is solved by using the forecasted load continuously to determine the optimal network topology with the minimum amount of loss at the future time. The simulation results validate and evaluate the proposed approach.
\end{abstract}

Index Terms - Short-term load forecasting, parallel parameters optimization, network reconfiguration, distribution system.

\section{INTRODUCTION}

$\mathrm{N}_{\mathrm{t}}^{\mathrm{t}}$ ETWORK reconfiguration alters a distribution network topology to improve the distribution system performance by opening sectionalizing switches (which are normally closed) and closing tie switches (which are normally open) without violating system operational constraints; this is mainly used to reduce energy loss and improve voltage profiles [1], [2]. A traditional distribution network reconfiguration is a one-time operation to achieve the minimum amount of loss by reconfiguring the network from one topology to another; however, with increasing penetrations of renewable energy resources and demand response programs, the end user profiles become more dynamic, which bring increasingly stochastic deviations in load profiles and bigger challenges in distribution system reconfiguration [2]-[5]. If a distribution load profile can be forecasted, the dynamic distribution system reconfiguration can be more effective and efficient. In this paper, the aggregated loads of a small section of a distribution system are forecasted by a load forecasting approach based on support

H. Jiang (email: Huaiguang.Jiang@nrel.gov), F. Ding, and Y. Zhang are with National Renewable Energy Laboratory (NREL), Golden, CO 80214 USA. This work was supported by the U.S. Department of Energy under Contract No. DE-AC36-08GO28308 with the National Renewable Energy Laboratory. vector regression (SVR) to provide useful information for the distribution system reconfiguration.

Specifically, in SVR the parameters optimization is a critical problem, which decides the effectiveness and accuracy of the forecaster. Particle swarm optimization (PSO) is an effective method for parameters optimization; however, it is hard to guarantee that the PSO can converge to a global optimization [6], [7]; thus, a combined grid traverse algorithm (GTA) and $\mathrm{PSO}$, a two-step parameters optimization approach, is designed to solve this problem.

Based on the Hadoop, Spark, and many other parallel computation frameworks, a work note can be a computer in a cluster or a single computer processing core (CPU) core in a multicore CPU [8], [9]. In this paper, a parallel computation architecture based on "Mapreduce" [15] is designed to further reduce the computation time in the two-step parameter optimization approach. Then the forecasted high-resolution loads are used for the network reconfiguration.

Although a network reconfiguration is a complicated nonconvex and nonlinear optimization problem, the optimal topology with the minimum amount of loss for a specific system is indeed determined by its net load profile. If the net load profile keeps changing, the optimal topology should be solved again to achieve the minimum loss under the new profile. Thus, with the forecasted distribution load profiles, the dynamic network reconfiguration can be pre-performed in response to load changes. Compared to reconfiguring the network by following the fixed time schedule, this data-driven reconfiguration is much more efficient and meaningful because it provides more observability to the distribution system operator (DSO) to determine the best system topology before an event happens. Also, it helps avoid unnecessary reconfiguration operations and captures the load dynamics and thus leads to more energy savings.

\section{SVR-BASED FORECASTER}

In this paper, the historic load data is used as input data to train the SVR-based forecaster. Considering the inconsistent, incomplete, and out-of-range values in the raw data, the data preprocessing methods are used as the first step for the input data [4], [9], [11].

In [10], the objective function of the SVR is defined to minimize the forecast error with coefficients regulation as follows:

$$
R=\min _{\varepsilon, \omega, \epsilon_{i^{\prime \prime}}, \epsilon_{i^{\prime \prime}}^{*} \mathrm{C}, \mathrm{b}, \gamma}\left\{\frac{1}{2} \omega^{T} \omega+\mathrm{C} \sum_{i^{\prime \prime}=1}^{n}\left(\epsilon_{i^{\prime \prime}}+\epsilon_{i^{\prime \prime}}^{*}\right)\right\}
$$


subject to:

$$
\left\{\begin{aligned}
L_{i^{\prime \prime}}-f\left(x_{i^{\prime \prime}}\right) & \leq \varepsilon+\epsilon_{i^{\prime \prime}} \\
-L_{i^{\prime \prime}}+f\left(x_{i^{\prime \prime}}\right) & \leq \varepsilon+\epsilon_{i^{\prime \prime}}^{*} \\
\epsilon_{i^{\prime \prime}}, \epsilon_{i^{\prime \prime}}^{*} & \geq 0
\end{aligned}\right.
$$

where $\frac{1}{2} \omega^{T} \omega$ indicates the coefficient flatness; $C$ is a trade-off coefficient; $\gamma$ is a coefficient in kernel; and $\epsilon_{i^{\prime \prime}}$ and $\epsilon_{i^{\prime \prime}}^{*}$ are two slack variables, which indicate the training error thresholds. In [10], the parameters $C, \omega$, and $\gamma$ are chosen as optimal variables, which play important roles in SVR performance. Then the objection function (1) with constraint (2) can be solved, and the parameters $\omega, C, \epsilon_{i^{\prime \prime}}$, and $\epsilon_{i^{\prime \prime}}^{*}$ can be determined.

\section{TWO-STEP PARALLEL PARAMETERS OPTIMIZATION}

\section{A. Architecture of the Approach}

As shown in Fig. 1, the proposed parameter optimization approach consists of two components: GTA and PSO. Specifically, in the first step, a GTA is designed to divide the global solution space into local solution spaces, and traverse the local solution spaces. In the Map phase, considering the independency among the local solution spaces, each can be mapped into a group of work notes and computed in parallel. Then in the Reduce phase, the computation results are compared, and one or several local solution spaces with minimum training errors are selected as the sub-local solution spaces for the PSO. In the second step, a PSO is used to optimize the parameters in the sub-local solution spaces, which also contain the Map and Reduce phases for the parallel computation. Finally, the optimal parameters can be determined effectively for the SVR-based forecaster.

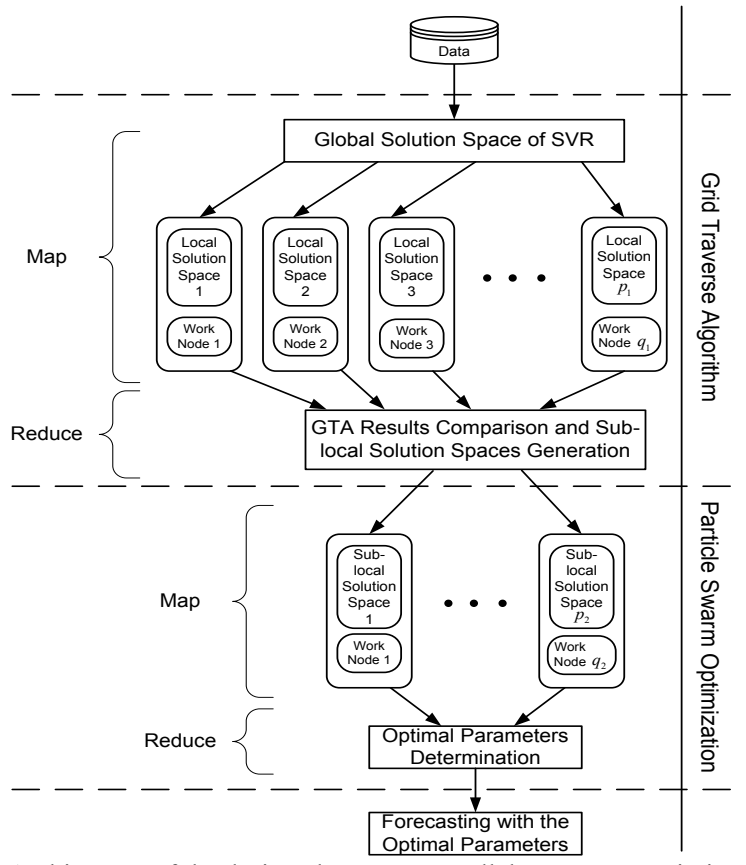

Fig. 1. Architecture of the designed two-step parallel parameter optimization approach

\section{B. Grid Traverse Algorithm}

As shown in Fig. 1, in the GTA the global solution space is divided into several local solution spaces. For each parameter
$\Omega_{j_{1}}$, a traversing vector $\Lambda_{j_{1}}$ can be built with corresponding step $\Delta_{j_{1}}$. For example, the $m_{j}^{\prime \prime}$ th element in $\Lambda_{j_{1}}$ can be built as:

$$
\Lambda_{j_{1}}^{m_{j_{1}}^{\prime \prime}}=\underline{\Omega_{j_{1}}}+\left(m_{j}^{\prime \prime}-1\right) \frac{\Omega_{j_{1}}-\overline{\Omega_{j_{1}}}}{\Delta_{j_{1}}}
$$

where $\Omega_{j_{1}}$ and $\overline{\Omega_{j_{1}}}$ are the upper and lower limits. Then a finite, multi-Cartesian product $\boldsymbol{H}$ can be generated with every traversing vector $\Lambda_{j_{1}}$.

In this paper, three parameters $-C, \omega$, and $\gamma$-are chosen, and the total number of the elements in $\boldsymbol{H}$ is $m_{1} \times m_{2} \times m_{3}$. This means that the global solution space can be divided into four local solution spaces, and each can be mapped to a different work note and computed in parallel. In addition, the 10 -fold cross-validation is used to limit overfitting in forecasting [10].

As shown in Algorithm 1, the GTA is targeted to decompose the global solution space and select one or several local solution spaces with minimum training errors for the next PSO processing. In the first step, besides the parameters and traversing vectors initialization, the work notes are initialized from an idle state. Specifically, for a computer cluster, the master can pick the idle computer. For a single computer with a multicore CPU, the idle cores are picked and assigned with memories. In the second step, every work note receives the assigned tasks and can compute in parallel. In the last step, the master collects all the results and selects one or several local solution spaces for the next PSO processing.

\section{Algorithm 1. Parallel GTA for Parameter Optimization}

Objective: Solution space decomposition and local solution spaces selection of the global optimization problem.

Initialization: (1) Initial $\boldsymbol{C}, \boldsymbol{\omega}$, and $\boldsymbol{\gamma}$, compute $\boldsymbol{\Lambda}_{\boldsymbol{j}}$ and $\boldsymbol{H}$.

(2) Initial the work notes.

Map phase: (1) Send the elements of $\boldsymbol{H}$ to all the work notes.

(2) In different work notes, the received elements of $\boldsymbol{H}$ are computed in parallel.

Reduce phase: (1) Collects computation results from all the work notes.

(2) Results comparison and one or several local solution spaces are selected for next step.

\section{Particle Swarm Optimization}

In the PSO, the number of the particles is $N_{P S O}$, and the dimension of the solution space is $n_{O B J P S O}=3$. Then the position of the $i_{2}$ th particle can be generated as $\alpha_{i_{2}}^{\Omega}=$ $\left[\alpha_{i_{2}, 1}^{\Omega} \alpha_{i_{2}, 1}^{\Omega} \ldots \alpha_{i_{2}, n_{O B J P S O}}^{\Omega}\right]$, where $\Omega=\left[\begin{array}{lll}C & \omega & \gamma\end{array}\right]$. Then the vector of velocity $v_{i_{2}}^{\Omega}$ and best position $\eta_{i_{2}}^{\Omega}$ of the $i_{2}$ th particle can be generated similarly.

In each iteration, the velocity and position can be determined as follows:

$$
\begin{aligned}
v_{i_{2}}^{\Omega}(\mathrm{t})= & v_{i_{2}}^{\Omega}(\mathrm{t}-1)+\varphi_{1} \theta_{1}\left(\eta_{i_{2}}^{\Omega}-\alpha_{i_{2}}^{\Omega}(t-1)\right) \\
& +\varphi_{2} \theta_{2}\left(\eta_{g}^{\Omega}-\alpha_{i_{2}}^{\Omega}(t-1)\right)
\end{aligned}
$$




$$
\alpha_{i_{2}}^{\Omega}(t)=\alpha_{i_{2}}^{\Omega}(t-1)+v_{i_{2}}^{\Omega}(\mathrm{t})
$$

where the independently distribution random variables are $\theta_{1}$ and $\theta_{2}$, the acceleration coefficients are $\varphi_{1}$ and $\varphi_{2}, t$ is the time index, and the best position vector is $\eta_{g}^{\Omega}$. Thus, as shown in Fig. 1, the selected sub-local solution spaces can be computed with (4) and (5) in parallel, and the best parameters can be determined.

\section{A Short-Term Load Forecast Example}

A short-term load forecast for a distribution feeder is shown in Fig. 2. The blue curve indicates the original load profile, and the red curve indicates the forecasted load profile. From 1$14,400 \mathrm{~s}$ is the training data, and from $14,401-18,000 \mathrm{~s}$ is the forecast result, which is defined as 1-h-ahead forecasting. On the one hand, the original load profile indicates that the distribution system loads contain more stochastic abrupt deviations than the transmission system loads. On the other hand, the forecasted load profile is almost consistent with the original load profile, which demonstrates the effectiveness and efficiency of the proposed approach.

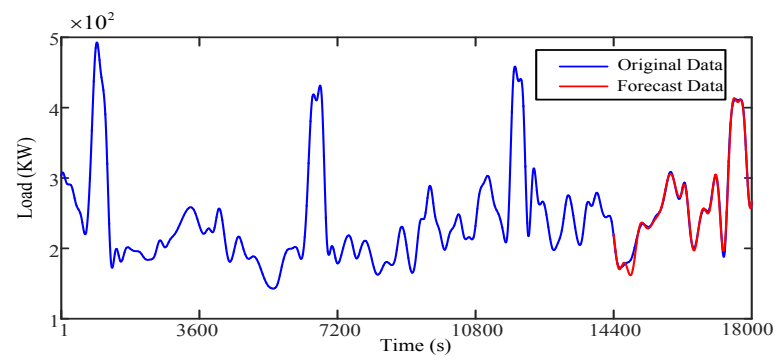

Fig. 2. Forecast result of a distribution system load

\section{ShORT-TERm LOAD ForeCASTING NETWORK RECONFIGURATION STUDY}

\section{A. Network Reconfiguration Optimization Problem}

In a distribution system, the state of each controllable switch is defined as:

$$
S_{j}=\left\{\begin{array}{l}
1, \text { switch } j \text { is closed and current direction keeps same } \\
-1, \text { switch } j \text { is closed and current direction is opposite. } \\
0, \text { switch } j \text { is open. }
\end{array}\right.
$$

A node-branch incidence matrix is used to define the connectivity of a network. Suppose all controllable switches are closed, and the node-branch incidence matrix for the meshed network is denoted as $\mathbf{A}^{\mathbf{0}}$. For any other system topology after opening/closing the switches, the new node-branch incidence matrix, denoted as $\mathbf{A}$, can be derived from $\mathbf{A}^{\mathbf{0}}$ using the states of switches. This paper takes use of network reconfiguration to minimize the power loss, so that finally the optimization problem can be defined as shown below. (For more details, see [2].)

$$
\begin{aligned}
& \min J(S)=\operatorname{Re}\left\{\sum_{j=1}^{N}\left[\sum_{k=a}^{c} V_{j}^{*} \cdot \sum_{i=1}^{N}\left(\sum_{p=a}^{c} V_{i}^{p} \cdot\left(\sum_{l=1}^{m} a_{I}^{0} a_{j j}^{0} \nu_{l}^{p k} \cdot S_{l}^{2}\right)\right)\right]\right\} \\
& =\sum_{j=1}^{N} \sum_{k=a}^{c} \sum_{i=1}^{N} \sum_{p=a}^{c}\left\{e_{\bar{j}}^{k} \cdot\left(e_{\bar{i}}^{p} \cdot g_{\bar{y}}^{p k}+f_{\bar{i}}^{p} \cdot b_{\bar{y}}^{p k}\right)+f_{j}^{k} \cdot\left(f_{\bar{i}}^{p} \cdot g_{i \bar{j}}^{p k}-e_{\bar{i}}^{p} \cdot b_{\bar{y}}^{p k}\right)\right\} \\
& \text { s.t. }\left\{\begin{array}{l}
P_{\text {inject }, i}^{l}=\sum_{k=1}^{n} \sum_{p=a}^{c}\left\{e_{i}^{l}\left(g_{i k}^{l p} \cdot e_{k}^{p}-b_{i k}^{l p} \cdot f_{k}^{p}\right)+f_{i}^{l}\left(g_{i k}^{l p} \cdot f_{k}^{p}+b_{i k}^{l p} \cdot e_{k}^{p}\right)\right\} \\
Q_{i n j e c t, i}^{l}=\sum_{k=1}^{n} \sum_{p=a}^{c}\left\{f_{i}^{l}\left(g_{i k}^{l p} \cdot e_{k}^{p}-b_{i k}^{l p} \cdot f_{k}^{p}\right)-e_{i}^{l}\left(g_{i k}^{l p} \cdot f_{k}^{p}+b_{i k}^{l p} \cdot e_{k}^{p}\right)\right\}
\end{array}\right. \\
& \left\{\begin{array}{l}
0.95 \cdot\left|V_{\text {norm }}\right| \leq\left|V_{i}^{a}\right|,\left|V_{i}^{b}\right|,\left|V_{i}^{c}\right| \leq 1.05 \cdot\left|V_{\text {norm }}\right| \\
\left|\begin{array}{l}
V_{i}^{p}\left|-\operatorname{avg}_{i}\right| \\
\operatorname{avg}_{i}
\end{array}\right| \leq 3 \%, \text { and } \operatorname{avg}_{i}=\sum_{p=a}^{c}\left|V_{i}^{p}\right| / 3 \\
\left|I_{\text {branch }, j}^{p}\right| \leq I_{j, \max } \\
i=1,2, \ldots, N ; j=1,2, \ldots, M ; l=a, b, c ; p=a, b, c .
\end{array}\right.
\end{aligned}
$$

where $a_{i j}=a_{i j}^{0} \cdot S_{j}, a_{\mathrm{ij}}{ }^{0}$ are the $i j$-th elements of $\mathbf{A}$ and $\mathbf{A}^{\mathbf{0}} . N$ and $M$ are the total number of buses and the total number of branches, respectively. $P_{\text {inject }, i}^{p}$ and $Q_{\text {inject }, i}^{p}$ are the actual phase- $p$ ( $p$ can be $a, b, c$.) active and reactive power injections at bus $i . V_{i}^{p}=e_{i}^{p}+\mathrm{j} f_{i}^{p}$ is the phase- $p$ voltage at bus $i . I_{\text {branch }, j}^{p}$ is the phase- $p$ current in branch $j . I_{j}^{\max }$ is the maximum current allowed for branch $j$. Matrix $\left[y_{j}^{a a}, y_{j}^{a b}, y_{j}^{a c} ; y_{j}^{b a}, y_{j}^{b b}, y_{j}^{b c} ; y_{j}^{c a}, y_{j}^{c b}, y_{j}^{c c}\right]$ is the conjugate inverse matrix of the phase impedance matrix for branch $j$. And $g_{i k}^{l p}+j b_{i k}^{l p}=\sum_{j=1}^{M}\left(a_{i j}^{0} a_{k j}^{0} y_{j}^{l p}\right) \cdot S_{j}^{2}$.

The voltage and thermal constraints are included in (7). In addition, the system topology after the reconfiguration must be radial without meshes, and all loads must be served without disconnection. These constraints are formulated as:

$$
\left\{\begin{array}{l}
\sum_{k=1}^{M}\left|S_{k}\right|=N-d \text { and } \sum_{i=1}^{M_{k}}\left|S_{i}\right| \leq M_{k}-1 \\
\operatorname{rank}(A)=N-d
\end{array}\right.
$$

where $d$ is the total number of slack buses, and $M_{k}$ is the total number of branches in loop $k$. As a result, the network reconfiguration problem is finally formulated as a mixed-integer nonlinear optimization problem with switch states as the only decision variable.

When using the high-resolution load data, the dynamic network reconfiguration problem will be solved frequently, so a highly computationally efficient algorithm is preferred; thus, this paper uses the heuristic algorithm based on the branch-exchange and single-loop optimization to solve the network reconfiguration algorithm. (For the flowchart of the algorithm, see [1].) 


\section{B. Data-Driven Network Reconfiguration Based on Load Forecasting}

Fig. 3 shows the framework of the proposed data-driven network reconfiguration based on the short-term load forecasting. At first, the short-term load forecasting is executed using the historic actual high-resolution data. This paper studies the 1-h ahead forecasting using the 1-s resolution data. Next, the network reconfiguration problem defined in (7) and (8) is solved using the heuristic algorithm continuously and the forecasted next-hour load data, and the highest resolution is to solve the reconfiguration problem using every second of data, leading to 3,600 results. However, considering that the load change happened in $1 \mathrm{~s}$ is generally not big enough to alter the optimal topology, so the reconfiguration problem is solved for every $5 \mathrm{~min}$ in this paper, finally leading to 12 results of the system topology for the next hour and each result for a 5-min time slot. Among all 12 system topologies for the 5-min time slots, the topology that achieves the most loss reduction will be selected and used for the entire next hour. This helps reduce the reconfiguration operation compared to using one topology every 5 minute.

Then the above procedures will be repeated for the following hours. Compared to the traditional dynamic network reconfiguration, which is based on the fixed time schedule without load forecasting, the proposed data-driven network reconfiguration based on short-term load forecasting enables the DSO to observe the events that will happen in the next hour and make decisions based on all future events; thus, it is highly likely to achieve better performance.

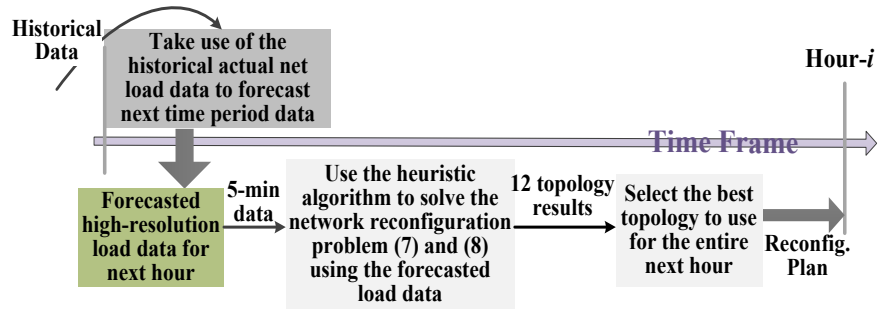

Fig. 3. Data-driven network reconfiguration approach based on short-term load forecasting

\section{CAse Study}

The proposed data-driven network reconfiguration approach based on short-term load forecasting is tested on a revised version of the IEEE 123-bus distribution system. As shown in Fig. 4, compared to the benchmark IEEE 123-bus test system given in [3], [16], four initially opened tie switches (TS-1, TS-2, TS-3 and TS-4) are added to make the system topology changeable, and all voltage regulators are removed to fully address the impact of network reconfiguration on reducing losses.

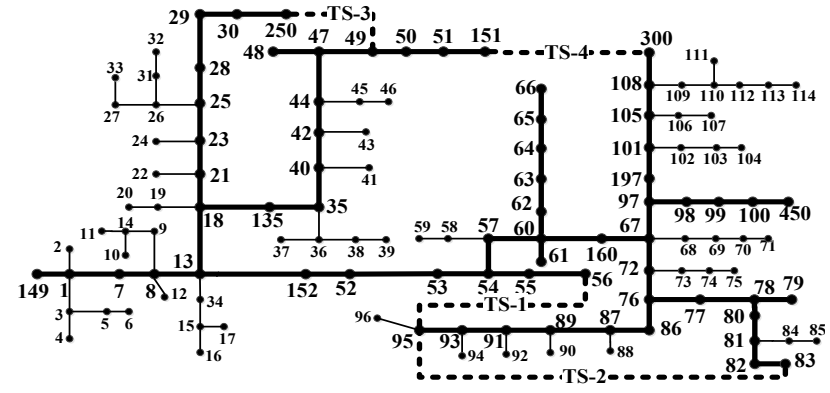

Fig. 4. Configuration of the revised IEEE 123-bus test system

\section{A. Short-Term Load Forecasting}

As shown in Fig. 5, the four net load profiles are forecasted by the proposed 1-h-ahead forecasting approach with 1-s resolution. The tests load data set consists of four net load profiles from a partner utility's distribution feeder. In this paper, the net load profile includes both loads and distributed energy resources (DERs) so the net load can be negative when the DER generation exceeds the load demand.

The forecast error is shown in Fig. 6. Note that more than $80 \%$ of the errors are accumulated between $(-3 \%, 3 \%)$. The MAPE is $2.15 \%$, and NRMSE is $4.03 \%$. In addition, the skewness and kurtosis are 0.71 and 7.23 , which are used to measure the asymmetry and outlier prone of the probability distribution of the forecast errors [13], [14], [17], [18]. The average computation time is $72 \mathrm{~s}$, with an Intel Xeon CPU Server. The forecast error analysis demonstrates the accuracy and effectiveness of the proposed distribution net load forecasting approach.

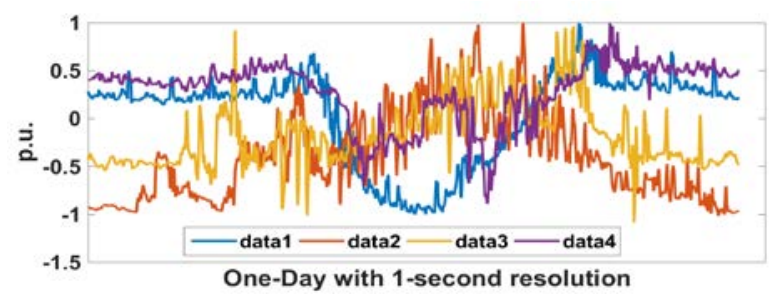

Fig. 5. Four profiles of forecasted net loads for four different regions

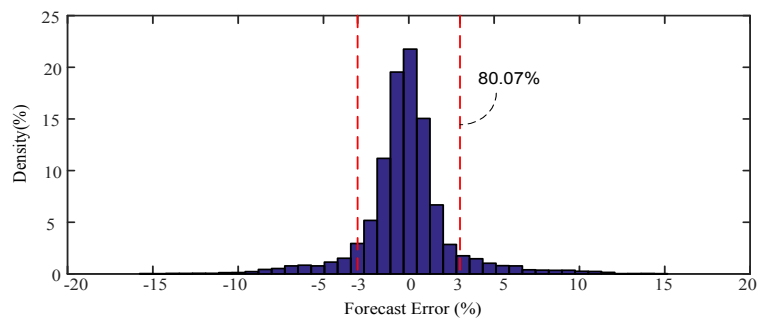

Fig. 6. Percentage error of forecast results

\section{B. Reconfiguration Results}

The benefit of the proposed approach contains two parts: total energy loss reduction and network reconfiguration operation reduction, which can be explained as follows. In Fig. 3, the data-driven network reconfiguration based on the 1-h ahead load forecasting is solved. Table III lists the results of the opened switches for each 5-min time slot within each hour. If multiple consecutive time slots have the same topology result, their results are aggregated to be displayed. The power loss 
reduction achieved by each topology change is also listed. Among all topology results within $1 \mathrm{~h}$, the topology that leads to the largest energy loss reduction (the product of power loss reduction and time duration) is selected as the final topology for the entire hour, which is highlighted using the color block.

Evaluating all final topology results for $24 \mathrm{~h}, 7$ reconfiguration operations will be implemented, including: (1) opening switches at lines 93-95, 29-30, 101-105 and closing switches TS-1, TS-3, and TS-4 at 1 a.m.; (2) opening switches 87-89 and 108-300 and closing switches $93-95$ and 101-105 at 6 a.m.; (3) opening switch 57-60 and closing switch 108-300 at 8 a.m.; (4) opening switch 67-72 and closing switch 87-89 at 9 a.m.; (5) opening switch 18-21 and closing switch $29-30$ at 10 a.m.; (6) opening switch 29-30 and closing switch 18-21 at 3 p.m.; (7) opening switch 18-21 and closing switch $29-30$ at 5 p.m.

TABLE III

RESULTS OF OPEN SWITCHES SOLVED USING 5-MIN FORECASTED LOAD

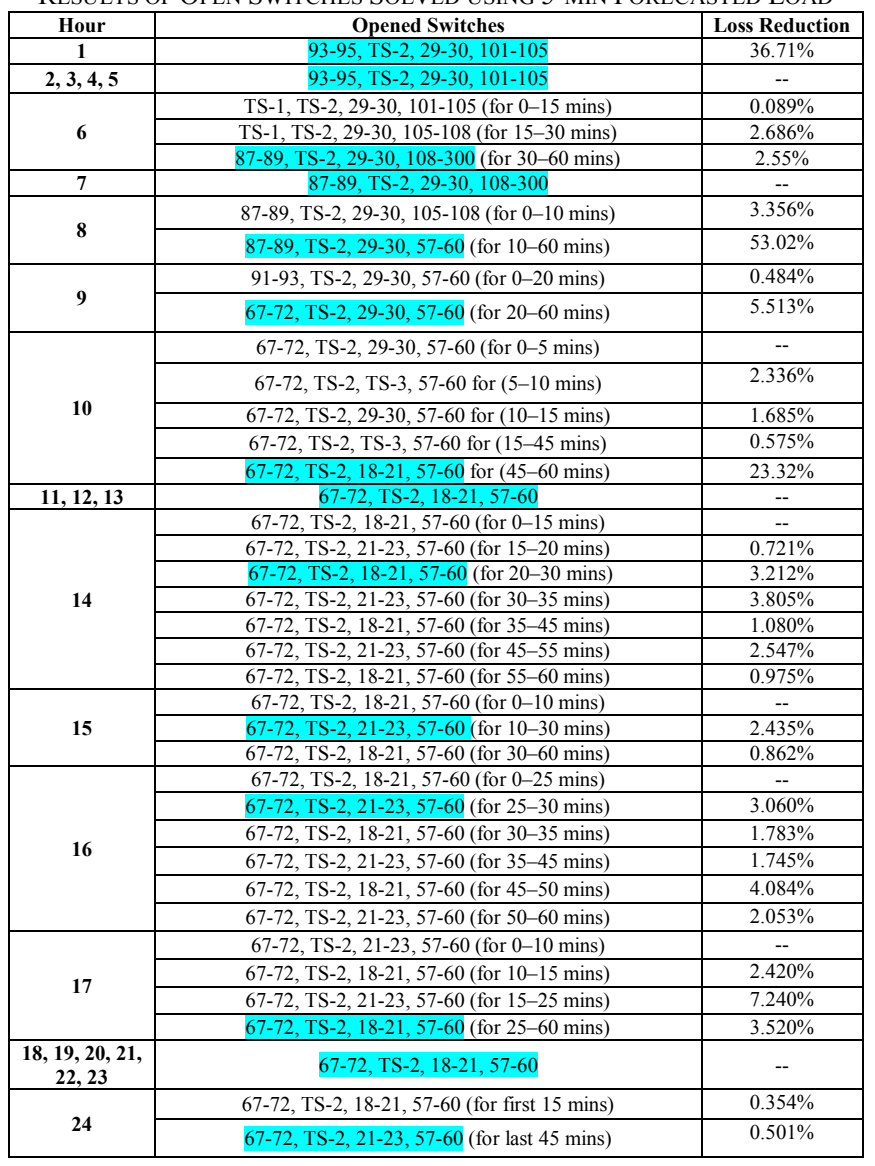

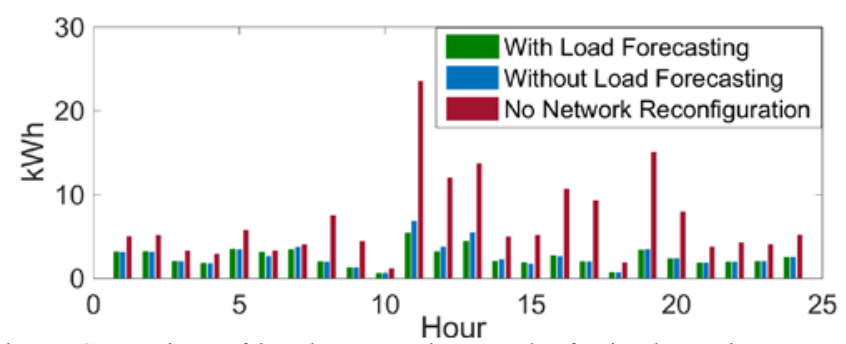

Fig. 7. Comparison of hourly energy loss results for implementing network reconfiguration with load forecasting, implementing network reconfiguration without load forecasting, and no network reconfiguration
As shown in Fig. 7, the hourly energy loss is obtained, and it is compared to the results obtained using the fixed hourly reconfiguration without load forecasting and for the no reconfiguration study.

The benefit of the proposed approach is twofold: total energy loss reduction and network reconfiguration operation reduction. The total energy losses in a day for these three scenarios are $60.49 \mathbf{~ k W h}$ (with load forecasting), $71.81 \mathrm{kWh}$ (without load forecasting), and $164.23 \mathrm{kWh}$ (no network reconfiguration), respectively. In addition, the optimal topologies solved using the network reconfiguration with a fixed hourly time schedule but without load forecasting get changed at 1 a.m., 7 a.m., 8 a.m., 10 a.m., 11 a.m., 1 p.m., 2 p.m., 3 p.m., 4 p.m., 5 p.m., 6 p.m., 7 p.m., 8 p.m., 9 p.m., and 11 p.m.; thus, a total of $\mathbf{1 5}$ reconfiguration operations occur in a day, which is an increase of $\mathbf{8}$ reconfiguration operations than the proposed approach with load forecasting. In summary, compared to the traditional network reconfiguration approach, the proposed approach reduces system energy loss by approximately $15 \%$ and network reconfiguration operations by $\mathbf{5 0 \%}$.

\section{CONCLUSIONS AND Future WORK}

Compared to the traditional network reconfiguration approach, a novel distribution system network reconfiguration based on short-term load forecasting is proposed for a system with a high penetration of renewable energy. According to the case study, the net load profiles can be accurately forecasted with the proposed forecasting approach. Then, by using the 1-h-ahead forecasted load data to predetermine the optimal topology for the next hour, the system energy loss can be reduced with a reduced number of switching operations compared to the fixed scheduled network reconfiguration approach without load forecasting. Thus, this paper has proven the feasibility and effectiveness of the proposed reconfiguration approach.

In the next step, different types of events such as unplanned islanding and renewable generation intermittency will be studied.

\section{REFERENCES}

[1] F. Ding and K. A. Loparo, "Hierarchical decentralized network reconfiguration for smart distribution systems-Part I: Problem formulation and algorithm development," IEEE Trans. Power Syst., vol. 30, no. 2, pp. 734-743, March 2015.

[2] F. Ding and K. A. Loparo, "Feeder reconfiguration for unbalanced distribution systems with distributed generation: A hierarchical decentralized approach," IEEE Trans. Power Syst.ems, vol. 31, no. 2, pp. 1,633-1,642, March 2016.

[3] Distribution System Analysis Subcommittee Report, "Radial Distribution Test Feeders," http://www.ewh.ieee.org/soc/pes/dsacom/ testfeeders/index.html.

[4] H. Jiang, Y. Zhang, J. J. Zhang, D. W. Gao, and E. Muljadi, "Synchrophasor-based auxiliary controller to enhance the voltage stability of a distribution system with high renewable energy penetration," IEEE Trans. Smart Grid, vol. 6, no. 4, pp. 2,107-2,115, 2015.

[5] H. Jiang, X. Dai, W. Gao, J. J. Zhang, Y. Zhang, and E. Muljadi, "Spatial-temporal synchrophasor data characterization and analytics in smart grid fault detection, identification and impact causal analysis," IEEE Trans. Smart Grid, vol. 7, no. 5, pp. 2,525-2,536. 
[6] C. Wang, Y. Li, K. Peng, B. Hong, Z. Wu, and C. Sun, "Coordinated optimal design of inverter controllers in a micro-grid with multiple distributed generation units," IEEE Trans. Power Syst., vol. 28, no. 3, pp. 2,679-2,687, Aug. 2013.

[7] F. Van Den Bergh, "An analysis of particle swarm optimizers," Ph.D. dissertation, University of Pretoria, 2006.

[8] M. Zaharia, M. Chowdhury, M. J. Franklin, S. Shenker, and I. Stoica, "Spark: Cluster computing with working sets," HotCloud, vol. 10, pp. $10-10,2010$.

[9] Y. Gu, H. Jiang, Y. Zhang, J. J. Zhang, T. Gao, E. Muljadi, "Knowledge discovery for smart grid operation, control, and situation awareness - a big data visualization platform," North American Power Symp. (NAPS), 2016, pp. 1-6.

[10] C.-C. Chang and C.-J. Lin, "Libsvm: A library for support vector machines," ACM Transactions on Intelligent Systems and Technology (TIST), vol. 2, no. 3, p. 27, 2011.

[11] H. Jiang, Y. Zhang, E. Muljadi, J. J. Zhang, D. W. Gao, “A short-term and high-resolution distribution system load forecasting approach using support vector regression with hybrid parameters optimization," IEEE Trans. Smart Grid, to be published.

[12] William H. Kersting, Distribution System Modeling and Analysis. CRC Press, 2001.

[13] K. V. Mardia, "Measures of multivariate skewness and kurtosis with applications," Biometrika, vol. 57, no. 3, pp. 519-530, 1970.

[14] E. Jondeau and M. Rockinger, "Conditional volatility, skewness, and kurtosis: existence, persistence, and comovements," J. Economic Dynamics and Control, vol. 27, no. 10, pp. 1,699-1,737, 2003.

[15] J. Dean and S. Ghemawat, "Mapreduce: Simplified data processing on large clusters," Communications of the ACM, vol. 51, no. 1, pp. 107-113, 2008.

[16] F. Ding, H. Jiang, and J. Tan, "Automatic distribution network reconfiguration: An event-driven approach," in Power and Energy Society General Meeting (PESGM), 2016. IEEE, 2016, pp. 1-5.

[17] H. Wu, M Shahidehpour, ME Khodayar, "Hourly demand response in day-ahead scheduling considering generating unit ramping cost," IEEE Trans. Power Syst., vol. 28, no. 3, pp. 2446-2454, Aug. 2013.

[18] H. Jiang and Y. Zhang, "Short-term distribution system state forecast based on optimal synchrophasor sensor placement and extreme learning machine," in Power and Energy Society General Meeting (PESGM), 2016. IEEE, 2016, pp. 1-5. 